\title{
Complexo Econômico-Industrial da Saúde
}

\section{Economic-Industrial Health Care Complex}

\section{Complejo Económico-Industrial de la Salud}

Everton Nunes da SILVA ${ }^{1}$

Faculdade de Ceilândia - FCE/UnB

Centro Metropolitano - Conjunto A - Lote 01

Brasília/DF - CEP: 72220-900

Telefone: +55 (61) 3107-8400

evertonsilva@unb.br

O autor declara não haver potenciais conflitos de interesse.

Resumo: O objetivo deste artigo é apontar a importância do CEIS tanto em termos sociossanitário quanto econômico. Ademais, pretende-se apresentar as principais medidas adotadas pelo governo federal para fomentar o CEIS, além de identificar alguns desafios que ainda persistem nessa área. Um CEIS nacional, associado com os estímulos e a regulamentação do Estado, tende a: i) estar mais próximo das necessidades em saúde da população; ii) fixar internamente recursos humanos de elevado capital humano; iii) ter efeitos positivos na renda interna, pela criação de empregos e geração de renda; iv) reduzir o déficit comercial crônico na balança comercial na rubrica da saúde; v) mitigar a dependência científica, visto que geralmente não há transferência tecnológica do exterior para instituições brasileiras. As políticas públicas adotadas pelo governo federal evidenciam que há um esforço articulado para fomentar o CEIS do país, a exemplo da Política Industrial, Tecnológica e de Comércio Exterior (2003), Política de Desenvolvimento Produtivo (2008), Plano Brasil Maior (2011), Decreto n. 7.546/11 entre outras. A despeito das políticas adotadas até então, permanecem dois desafios no âmbito do CEIS: i) padrão de inserção produtiva no cenário mundial e na divisão internacional do trabalho; ii) e baixa geração de conhecimento com pouca densidade de pesquisa e desenvolvimento.

Palavras-chave: Complexo industrial da saúde; Economia da saúde; Inovação em saúde; Indústrias da saúde; Saúde e Desenvolvimento

\begin{abstract}
The purpose of this article is to point out the importance of the HIC both in economic and social-sanitary fields. Moreover, we intend to present the main measures adopted by the government to foster it, and identify some challenges that still exist in this area. A national HIC, associated with the stimuli and state regulations, tends to: i) be closer to the health needs of the population; ii) establish internal human resources with high human capital; iii) have positive effects on domestic income, by creating employment and income generation; iv) reduce the chronic trade deficit in the trade balance under health and v) mitigate the scientific dependence, since

1 Economista (PUC/RS), mestre (UFPE) e doutor em economia (UFRGS). Professor da Universidade de Brasília, do Curso de Saúde Coletiva da Faculdade de Ceilândia.
\end{abstract}

ISSN 1982-8829

Tempus, actas de saúde colet, Brasília, 8(1), 71-78, mar, 2014// 
there is usually no technology transfer from abroad to Brazilian institutions. The policies adopted by the federal government show that there is a concerted effort to foster the HIC of the country, such as the Industrial, Technological and Foreign Trade Policy (2003), Productive Development Policy (2008), Greater Brazil Plan (2011), Decree n. 7.546/11, among others. Despite the policies adopted so far, two challenges remain within the HIC: i) standard productive involve-Complexo Econômico-Industrial da Saúde Economic-Industrial Health Care Complex Complejo EconómicoIndustrial de la Salud ment on the world scenario and in the international division of labor, ii) low knowledge generation with low density of research and development.

Key-words: Health industrial complex; Health economics; Health innovation system; Health industries; Health and Development

Resumen: El objetivo de este artículo es señalar la importancia del CEIS, tanto en lo económico como en lo socio-sanitario. Además, tenemos la intención de presentar las principales medidas adoptadas por el gobierno para fomentarla, e identificar algunos de los desafios que aún existen en esta área. Un CEIS nacional, associado con los estímulos y las regulaciones estatales, tiende a: i) estar más cerca de las necesidades de salud de la población; ii) establecer los recursos humanos internos con alto capital humano; iii) tener efectos positivos en el ingreso nacional, mediante la creación de empleo y generación de ingresos; iv) reducir el déficit comercial crónico de la balanza comercial en Salud y v) mitigar la dependencia cientifica, ya que generalmente no hay transferencia de tecnología desde el extranjero a las instituciones brasileñas. Las políticas adoptadas por el gobierno federal evidencian que hay un esfuerzo concertado para promover el CEIS del país, como la Política Industrial y Tecnológica de Comercio Exterior (2003), la Política de Desarrollo Productivo (2008), el Plan Brasil Mayor (2011), Decreto n. 7.546/11, entre otros. A pesar de las políticas adoptadas hasta ahora, dos desafios permanecen dentro del CEIS: i) el modelo de la inserción productiva en el escenario mundial y en la división internacional del trabajo, ii) la baja generación de conocimiento com poca densidad de investigación y desarrollo. Palabras clave: Complejo Industrial de la Salud; Economía de la Salud; la Innovación en Salud; Industrias de la Salud; Salud y Desarrollo.

\section{INTRODUÇÃO}

Promover, restabelecer e manter a saúde da população de determinada sociedade têm sido os objetivos perseguidos pelos principais sistemas de saúde ao redor do mundo. Essa concepção foi afirmada na Constituição Federal de 1988, na qual se garantiu o direito à saúde como um direito de cidadania ${ }^{1}$.

Para haver efetivação dessas funções, é necessário um conjunto amplo de recursos: monetários, humanos, tecnológicos e de inovação; todos convergindo às necessidades da população. Nesse contexto, insere-se o complexo econômico-industrial da saúde (CEIS), definido como atividades produtivas interligadas que mantêm relações intersetorias entre $\mathbf{s i}^{2}$. 
O CEIS comporta três subsistemas: as indústrias de base química e biotecnológica (fármacos, medicamentos, vacinas, hemoderivados, reagentes para diagnóstico); as indústrias de base mecânica, eletrônica e de materiais (equipamentos, próteses, órteses, materiais de consumo, dispositivos médicos); e o setor de serviços da saúde (hospitais, clínicas, laboratórios de diagnóstico)3. Os dois primeiros interagem entre si em termos de compra e venda de bens e serviços (insumos, conhecimento, tecnologia, inovação), no intuito de gerar produtos a serem ofertados ao setor de serviços da saúde. Nessa triangulação sistêmica, tanto a oferta quanto a demanda interagem constantemente e, a partir dessas relações, tem-se a configuração da assistência sanitária do país.

Cabe destacar também o papel do Estado nesse contexto, o qual pode criar condições favoráveis ou desfavoráveis à atuação do CEIS. Em perspectiva histórica, houve momentos de convergências entre os anseios de fortalecimento do CEIS e a atuação do Estado, bem como rupturas nessa relação. Desde os primórdios das primeiras políticas públicas da saúde, o CEIS fez-se presente, mesmo em conjunturas econômicas adversas, como, por exemplo, no início do período republicano, o qual foi caracterizado por um modelo agroexportador, centrado na produção de poucos produtos primários para consumo externo4. Mesmo nesse contexto, identificou-se a necessidade de desenvolver tecnologias para evitar epidemias - entraves ao crescimento econômico. Assim, em 1900, o governo criou o Instituto Soroterápico Federal - depois chamado Instituto Oswaldo Cruz -, com a finalidade de produzir vacinas e soros contra doenças epidêmicas ${ }^{5}$, além de outras ações de âmbito estadual (Butantan, por exemplo).

A partir de 1930 até meados da década de 1980, o modelo econômico foi alterado, optandose pelo nacional-desenvolvimentismo. A industrialização era vista como a força motriz do progresso. Assim, fortaleceram-se as indústrias nacionais, o mercado interno e a maior autonomia econômica e tecnológica em relação aos países desenvolvidos5. As políticas sociais tornaram-se um subproduto do modelo de desenvolvimento e a incorporação dos direitos sociais pelas diversas classes profissionais ocorreria de maneira fragmentada e diversificada, sendo mais efetivas àquelas mais organizadas e inseridas no processo de modernização econômica6 (trabalhadores formais que atuavam na indústria). Devido às frágeis bases de financiamento do nacional-desenvolvimentismo - políticas monetárias e fiscais benevolentes -, esse modelo apresentou problemas estruturais (endividamento, escassez de divisas, inflação).

Na década de 1990, a estabilidade econômica tornou- se o principal objetivo econômico dos governos que se seguiram, implementando medidas de cunho ortodoxo. O estímulo ao CEIS foi arrefecido, dada à orientação político-econômica à época de redução do tamanho do Estado. Ao mesmo tempo, institui-se o Sistema Único de Saúde - SUS $S^{7,8}$, que necessitaria de maior aporte de recursos para implementar seus princípios de universalidade, integralidade, gratuidade, descentralização e controle social, bem como tecnologias e inovações para aumentar a longevidade e a qualidade de vida da população.

A partir década de 2000, entretanto, tem havido indícios de uma nova fase do desenvolvimento 
nacional, na qual a saúde tem sido incorporada como eixo estratégico ${ }^{3}$. Nessa direção, o objetivo deste artigo é apontar a importância do CEIS tanto em termos sociais - garantida de uma oferta de tecnologias e de inovações adequada às necessidades nacionais de saúde - quanto econômico crescimento e desenvolvimento.

Ademais, pretende-se apresentar as principais medidas adotadas pelo governo federal para fomentar o CEIS, além de identificar alguns desafios que ainda persistem nessa área.

\section{DESENVOLVIMENTO}

\section{Saúde, desenvolvimento e complexo econômico- industrial da saúde}

A demanda por ações e serviços de saúde tem se ampliado ao longo dos anos, seja em países desenvolvidos seja em países em desenvolvimento9. Vários fatores têm contribuído para isto: transições demográfica (urbanização, envelhecimento) e epidemiológica (doenças crônicas), incorporação tecnológica (intensiva em P\&D), saúde estabelecida como um componente de proteção social (sistemas universais), entre outros. Tais condicionantes têm atribuído ao CEIS um componente estratégico para a garantida de uma oferta equânime de bens e serviços de saúde. Os itens que se seguem buscam sinalizar a importância do CEIS nessa conjuntura.

-A oferta de tecnologias em saúde é fundamental para a sustentabilidade de um sistema de saúde universal e integral como o brasileiro ${ }^{10}$. Cada país tem suas especificidades em relação à demanda de cuidados em saúde, as quais podem não surtir interesse econômico de indústrias estrangeiras, como é o caso das doenças negligenciadas ${ }^{11}$. Um CEIS nacional, associado com os estímulos e a regulamentação do Estado, estaria mais próximo das necessidades em saúde da população.

- O CEIS é intensivo em conhecimento e inovação, mostrando-se um campo estratégico para adicionar valor ao Produto Interno Bruto. Os esforços mundiais em P\&D são expressivos na área da saúde, representando cerca de $20 \%$ do total, comparáveis apenas aos investimentos de defesa militar9.

- O crescimento do CEIS tende a ter efeitos transcendentais na economia como um todo, principalmente em termos de absorção de recursos humanos de elevado capital humano, visto que as inovações em saúde são dependentes de pesquisas multidisciplinares (química, biologia, engenharia, informática, medicina) ${ }^{12}$. Ademais, criaria condições de fixação de mão de obra especializada no Brasil, bem como efeitos positivos na renda interna, pela criação de empregos e geração de renda.

- Há um déficit comercial crônico na balança comercial na rubrica da saúde13, que evidencia a fragilidade da base produtiva e tecnológica brasileira frente aos países desenvolvidos10. Essa dependência por produtos importados pode comprometer a execução de políticas em saúde em momentos críticos: escassez de divisas, preços proibitivos praticados internacionalmente, 
interrupção de fornecimento de medicamentos/ vacinas em momentos de epidemias mundiais de grande proporção.

- Além da dependência comercial, há também a dependência científica, visto que geralmente não há transferência tecnológica do exterior para instituições brasileiras. Especificamente no setor farmacêutico, os investimentos em P\&D feitos no Brasil pelas indústrias do setor privado somam apenas $0,32 \%$ do faturamento, aplicados geralmente em estudos clínicos, mais como uma estratégia de marketing que de inovação ${ }^{14}$.

1.2 Políticas para o desenvolvimento do Complexo Econômico-Industrial da Saúde Diante do exposto, o governo federal tem sinalizado a importância do CEIS do país como uma das condições necessárias para o desenvolvimento econômico sustentável e a efetivação dos princípios do SUS, por meio de políticas articuladas e transversais entre as várias pastas ministeriais. Abaixo, seguem algumas delas:

- Em 2003, foi lançada a Política Industrial, Tecnológica e de Comércio Exterior (PITCE), a qual priorizou o seguimento farmacêutico como polo de inovação e fomento setorial (nanotecnologia, biotecnologia e química fina $)^{12,16}$.

- Em 2004, aprovaram-se na $2^{\text {a }}$ Conferência Nacional de Ciência, Tecnologia e Inovação em Saúde a Política Nacional de Ciência, Tecnologia e Inovação em Saúde e a Agenda de Prioridades de Pesquisa em Saúde, ambas contemplando o CEIS ${ }^{14}$.

- Ainda em 2004, institucionalizou-se a articulação entre instituições de Ciência e Tecnologia e de Saúde, juntamente com o Banco Nacional de Desenvolvimento Econômico e Social (BNDES), no intuito de priorizar a produção de inovação em saúde, por meio de concessão de financiamento para a área farmacêutica (Profarma I). Esse programa foi ampliado em 2007 para englobar todo o complexo industrial da saúde (Profarma II) $)^{10,12}$.

- Em 2007, o Programa Mais Saúde (PAC da Saúde) introduziu o CEIS como eixo estratégico para o desenvolvimento da base produtiva e da inovação?

- Em 2008, foi publicada a Política de Desenvolvimento Produtivo (PDP), na qual se reconheceu o CEIS como uma das seis áreas estratégicas para o desenvolvimento nacional, devido ao seu elevado potencial de disseminação de conhecimento e inovação. Apesar de não estar mais em vigor, esta representou um passo importante para o reconhecimento do CEIS na estratégia de desenvolvimento nacional ${ }^{9}$.

- Concomitante ao PDP, criou-se o Grupo Executivo do Complexo Industrial da Saúde - Gecis, composto por 14 órgãos federais, com a atribuição, entre outras, de desenvolver e implantar, de forma integrada, o marco regulatório necessário para a concretização das estratégias e diretrizes previstas na (PITCE fase II) coordenada pelo Ministério do Desenvolvimento, Indústria e Comércio 
Exterior, no Programa Mais Saúde do Ministério da Saúde e no Plano de Ação 2007-2010, promovendo a articulação dos órgãos e entidades do Governo Federal, com vistas a viabilizar um ambiente econômico e institucional propício ao desenvolvimento do Complexo Industrial da Saúde ${ }^{15}$.

- Em 2011, o Plano Brasil Maior foi anunciado, seguindo as linhas de ação do PDP e da PITCE, ao evidenciar o CEIS entre os blocos produtivos de dimensões relevantes ${ }^{9,10}$.

- No intuito de fortalecer a indústria nacional e promover a substituição de importações de produtos e insumos em saúde, o Decreto n. $7.546 / 11$ prevê margem de preferência de até $25 \%$ para a compra de produtos nacionais em âmbito federa $1^{16}$.

- Seguem em curso também as Parcerias para o Desenvolvimento Produtivo, medidas tomadas pelo Ministério da Saúde para fortalecer a indústria nacional. Atualmente, há mais de trinta parcerias estabelecidas entre laboratórios públicos e privados. Um marco importante nesse contexto foi a produção nacional do efavirenz em 2009, via licenciamento compulsório ${ }^{16}$.

\section{CONCLUSÃO}

As políticas públicas adotadas pelo governo federal evidenciam que há um esforço articulado para fomentar o CEIS do país, não apenas como uma estratégia econômica, com fins de geração de emprego e renda, mas também sociossanitária, com vistas à oferta adequada de tecnologias baseadas nas reais necessidades de saúde da população, levando em consideração a redução das desigualdades sociais. Nessa configuração, a aparente dicotomia entre as políticas econômica e social que se fez presente na década de 1990 (redução do tamanho do Estado versus implementação da SUS) se desfaz, pois as duas caminham na mesma direção.

Assim, resgata-se o arcabouço teórico-político do nacional-desenvolvimentismo, no qual o governo tem papel fundamental para a melhoria do Bem-Estar da população.

A despeito das políticas adotadas até então, permanecem dois desafios no âmbito do CEIS. O primeiro refere-se ao padrão de inserção produtiva no cenário mundial e na divisão internacional do trabalho; nossa economia ainda é pouco competitiva em termos de bens e serviços de alto valor agregado, tendo nas commodities nosso principal componente competitivo. O segundo diz respeito à baixa geração de conhecimento com pouca densidade de pesquisa e desenvolvimento, provavelmente por ainda permanecer a cultura estritamente acadêmica nas universidades (voltadas para curiosidades científicas, sem vislumbrar aplicabilidade prática), bem como a estrutura normativa que regem a relação entre público e privado, afugentando a possibilidade de interação entre universidades e indústrias. 


\section{REFERÊNCIAS BIBLIOGRÁFICAS}

1. Fleury S, Ouverney AM. Política de saúde: uma política social. In.: Giovanella L, Escorel S, Lobato LVC, Noronha JC, Carvalho AI (org.). Políticas e Sistemas de Saúde no Brasil. Fiocruz, 2008, 23-64.

2. Gadelha CAG, Maldonado, JMSV. Complexo industrial da saúde: dinâmica de inovação no âmbito da saúde. In.: Giovanella L, Escorel S, Lobato LVC, Noronha JC, Carvalho AI (org.). Políticas e Sistemas de Saúde no Brasil. Fiocruz, 2008, 247-282.

3. Gadelha CAG, Costa LS, Maldonado, JMSV. O complexo Econômico-Insdustrial da Saúde e a dimensão social e econômica do desenvolvimento. Rev. Saúde Pública 2012; 46(Supl): 21-28.

4. Gremaud AP, Vasconcellos MAS, Tonedo Neto R. Economia Brasileira Contemporânea. São Paulo: Atlas, $7^{\text {a }}$ Edição, 2007.

5. Escorel S, Teixeira LA. História das políticas de saúde no Brasil de 1822 a 1963: do Império ao desenvolvimento populista. In.: Giovanella L, Escorel S, Lobato LVC, Noronha JC, Carvalho AI (org.). Políticas e Sistemas de Saúde no Brasil. Fiocruz, 2008, 333-384.

6. Viana, ALA; Ibanez; Ibañez N, Elias PEM. Saúde, Desenvolvimento e Território. Editora Hucitec, São Paulo, 2009.

7. Escorel S. História das políticas de saúde no Brasil de 1964 a 1990: do golpe militar à Reforma Sanitária. In.: Giovanella L, Escorel S, Lobato LVC, Noronha JC, Carvalho AI (org.). Políticas e Sistemas de Saúde no Brasil. Fiocruz, 2008, 385-434.

8. Noronha JC, Lima LD, Machado, CV. O Sistema Único de Saúde - SUS. In.: Giovanella L, Escorel S, Lobato LVC, Noronha JC, Carvalho AI (org.). Políticas e Sistemas de Saúde no Brasil. Fiocruz, 2008, 435-472.

9. Gadelha CAG, Maldonado J, Vargas M, Barbosa PR, Costa LS. A dinâmica do sistema produtivo da saúde: inovação e complexo econômico-industrial. Rio de Janeiro: Fiocruz, 2012. $221 \mathrm{p}$.

10. Fiocruz. A saúde no Brasil em 2030: diretrizes para a prospecção estratégica do sistema de saúde brasileiro. Rio de Janeiro: Fiocruz, 2012. 323 p.

11. Brasil. Ministério da Saúde. Departamento de Ciência e Tecnologia. Secretaria de Ciência, Tecnologia e Insumos Estratégicos. Doenças negligenciadas: estratégias do Ministério da Saúde. Rev. Saúde Pública 2010; 44(1):200-202.

12. Viana ALA, Elias PEM. Saúde e desenvolvimento. Ciênc. saúde coletiva [online]. 2007, 
13. IBGE. Conta-Satélite da saúde: 2007-2009. Contas Nacionais numero 37. Rio de Janeiro, 2012.

14. Brasil. Anais da $2^{a}$ Conferência Nacional de Ciência, Tecnologia e Inovação em Saúde. Brasília: Ministério da Saúde, 2005, 272 p.

15. Brasil. Decreto Presidencial de 12 de maio de 2008. Cria, no âmbito do Ministério da Saúde, o Grupo Executivo do Complexo Industrial da Saúde Disponível em: http://www.planalto.gov.br/ ccivil_03/_Ato2007-2010/2008/Dnn/Dnn11578.htm. Acesso em 9 de julho de 2013.

16. Gadelha CAG, Costa LS. Saúde e desenvolvimento no Brasil: avanços e desafios. Rev Saúde Pública, 2012; 46(supl):13-20.

Artigo apresentado em 12/01/14

Artigo aprovado em 20/03/14

Artigo publicado no sistema: 29/03/14 